\title{
Force Measurements From Myofibril to Filament
}

\author{
Steven Marston* \\ NHLI, Imperial College London, London, United Kingdom
}

Contractility, the generation of force and movement by molecular motors, is the hallmark of all muscles, including striated muscle. Contractility can be studied at every level of organization from a whole animal to single molecules. Measurements at sub-cellular level are particularly useful since, in the absence of the excitation-contraction coupling system, the properties of the contractile proteins can be directly investigated; revealing mechanistic details not accessible in intact muscle. Moreover, the conditions can be manipulated with ease, for instance changes in activator $\mathrm{Ca}^{2+}$, small molecule effector concentration or phosphorylation levels and introducing mutations. Subcellular methods can be successfully applied to frozen materials and generally require the smallest amount of tissue, thus greatly increasing the range of possible experiments compared with the study of intact muscle and cells. Whilst measurement of movement at the subcellular level is relatively simple, measurement of force is more challenging. This mini review will describe current methods for measuring force production at the subcellular level including single myofibril and single myofilament techniques.

Keywords: contractility, myofibril contractility, thick filament, thin filament, tension measurement

\section{INTRODUCTION}

The production of force and movement by molecular motors is the essence of contractility. To understand the basic mechanisms of contraction and $\mathrm{Ca}^{2+}$ regulation we need to isolate the contractile elements from the physiological Excitation-Contraction (EC) system and from other, mainly parallel, mechanical elements that are present in whole muscle.

Contractility is usually measured in intact small muscle strips or single myocytes where contractility is dependent on the EC coupling system and neural and humoral stimulation that act on the $\mathrm{Ca}^{2+}$-regulation of the contractile apparatus itself. Chemically skinned muscle offers a way to study $\mathrm{Ca}^{2+}$ regulation of contractility in isolation from EC coupling but retains confounding effects of parallel elasticity. Moreover, the usefulness of skinned fiber studies is limited by slow diffusion of solutes into the fiber that rules out dynamic measurements. We therefore need to study contractility at the subcellular level.

Contractility measurements at the sub cellular level in myofibrils and single filaments offer advantages that make their development worthwhile. Solution conditions are easy to manipulate and rapid (i.e., $10 \mathrm{~ms}$ ) solution changing is possible since the solution diffuses rapidly into the myofibril so that the kinetics of the contractile response to rapid concentration jumps may be measured. There is no limitation on the source material that can be used and the quantities required are very small; myofibrils and myofilaments can be isolated from small samples (i.e., $20 \mathrm{mg}$ ) of muscle or cells. Fresh or frozen tissue are equally good sources and post-translational modifications 
can be changed or proteins substituted prior to measurement. Finally, since all measurements are made under the microscope it is possible to combine force measurements with additional video microscopy techniques such as observing sarcomeric structure dynamics and the turnover of fluorescent substrates.

To extend force measurements from muscle to single myofibrils or even single filaments requires a number of technological advances that were exceptional in the 1990s when these methods were first developed but have now become mainstream. Nevertheless, handling tiny samples is still challenging and is far from routine: whilst it is now easy to measure small forces, the problem of how to attach myofibrils and filaments to the measuring device is the greatest obstacle to high throughput experimentation.

In this review I will describe the current methodologies for single myofibril and single filament force measurements. At the time of writing none of the techniques described here can be bought as turnkey systems; to do such studies it is necessary to be able to build your own apparatus.

The principal of all force measurements is that the contractile element is attached to probes at both ends such that length changes can be imposed and force may be measured. The critical requirements for such measurements is that the contractile element is firmly attached to the probes and that the tension sensing probe be sufficiently compliant to enable probe movement to be translated into a signal yet sufficiently rigid that the contractile element is essentially isometric. The methods developed usually involve glass microneedles, AFM cantilevers or a stiff optical trap as the sensor. Table $\mathbf{1}$ summarizes the various methods that have been published.

\section{SINGLE MYOFIBRILS}

Measurement of force in single myofibrils has become the main technique used to study contractility at the subcellular level. Iwazumi (1987) published the earliest studies on myofibril contraction based on an electromagnetic force transducer and this methodology was used by Friedman and Goldman (1996) with some success but low throughput. A more versatile technique for measuring force in single myofibrils, based on glass microneedles, was first described by Bartoo et al. (1993) and was developed by Colomo et al. (1997) for active force measurement and by Linke et al. (1997) for passive force measurement. The principle of using glass microneedles for force measurement was introduced by Kishino and Yanagida (1988). More recent accounts (Ayittey et al., 2009; Vikhorev et al., 2015) describe how to make and calibrate the bent microneedles capable of measuring force in the $0.1-0.3 \mu \mathrm{N}$ range typical of single myofibrils. The prepared cantilever force probes have a compliance of $2-17 \mathrm{~mm} / \mathrm{mN}$ when deflected by small forces pulling perpendicularly.

This methodology that has been adopted by a number of laboratories. An alternative measurement technique based on an AFM cantilever force detector produces broadly similar results (Stehle et al., 2002). The microneedle-based methodology was thoroughly documented by Vikhorev et al. (2015) see Figure 1.
The heart of the instrument is the two microneedles. Myofibrils attach direct to glass and just touching the needle to the end of a myofibril results in firm adherence. In order to maximize the surface of myofibril attached to the probes they are manipulated to increase the myofibril wrapping around the microneedles. The tension transducer microneedle is crucial; the probe needs to be made in such a way that it is compliant enough to give sufficient bending under tension to be accurately measured in a position detector such as a split photodiode detector, yet stiff enough to keep the contracting muscle near isometric.

These force measurement methods are both sensitive and accurate, with high time resolution and low noise. They can be combined with rapid manipulation of length and uniquely can be used with fast solution changing and video microscopy of the sarcomere pattern, thus enabling a wide range of protocols that are not possible with large muscle preparations.

Rapid solution changing is the most important advantage of force measurements in myofibrils. Colomo et al. (1998) introduced the moving double-barreled pipette method that is almost universally used (Figures 1E,F). The time for solution switch to complete is less than $10 \mathrm{~ms}$. Since the smallness of myofibrils means that there is no diffusional lag, the rates of crossbridge attachment on a $\mathrm{Ca}^{2+}$ jump can be measured and the time course of relaxation when $\mathrm{Ca}^{2+}$ is removed can be followed (Figure 2). The absence of diffusional lag can be demonstrated by measuring the rate of crossbridge attachment following a rapid release and re-stretch $-\mathrm{K}_{\text {tr }}$ (Tesi et al., 2002) which is found to equal the rate of tension increase on $\mathrm{Ca}^{2+}$ jump (Figure 3A). Rapid removal of $\mathrm{Ca}^{2+}$ in myofibrils has revealed a two phase relaxation process that could not be detected in skinned fibers even with caged EGTA (Johns et al., 1999). Multiple solution changes enable dose-response curves to be generated, for instance the $\mathrm{Ca}^{2+}$ activation curve (Figure 3B).

The basic measurement of myofibrillar contractility is the $\mathrm{Ca}^{2+}$ activation-relaxation cycle (Figure 2). Maximum isometric contraction is around $100 \mathrm{~N} / \mathrm{m}^{2}$. The contraction and relaxation transients represent fundamental processes of the contractile apparatus not measurable in larger preparations.

$\mathrm{k}_{\mathrm{ACT}}$ represents the cross-bridge turnover rate which is determined by the dynamic equilibrium between the apparent rates with which cross bridges enter and leave the force generating states. It is dependent on the rate at which thin filaments are switched on by $\mathrm{Ca}^{2+}$ and is thus $\mathrm{Ca}^{2+}$ dependent (Vikhorev et al., 2014). Relaxation following a rapid drop in $\left(\mathrm{Ca}^{2+}\right)$ occurs in two phases. This pattern of results is only seen in myofibrils due to the rapid diffusion of $\mathrm{Ca}^{2+}$ away from the very small contractile element. Relaxation is initiated by $\mathrm{Ca}^{2+}$ dissociating from troponin $\mathrm{C}$ coupled to the release of the C-terminus of troponin I from troponin $\mathrm{C}$ and its attachment to actin, where it blocks cross-bridge binding cooperatively. The initial slow decrease in the force trace, defined by $\mathrm{k}_{\mathrm{LIN}}$ and $t_{\text {LIN }}$, has been ascribed to the time taken for the occupancy of cycling cross-bridges to drop below the threshold for cooperative activation of the thin filament, and the subsequent rapidrelaxation phase corresponds to the detachment of the remaining cross-bridges. $t_{\text {LIN }}$ is shortened and $\mathrm{k}_{\text {LIN }}$ increased by high phosphate concentrations but lengthened by MgADP indicating 
TABLE 1 | Techniques for single myofibril and single filament force measurements.

\begin{tabular}{|c|c|c|}
\hline References & Attachment method & Force transducer \\
\hline \multicolumn{3}{|l|}{ Myofibrils } \\
\hline Bartoo et al., 1993 & Silicone adhesive (Dow 3145 RTV) & Glass microneedle with split diode detector \\
\hline Colomo et al., 1997 & Myofibrils adhered directly to glass & Glass microneedle with split diode detector \\
\hline Stehle et al., 2002 & Silicon adhesive (Dow 3140 RTV) & $\begin{array}{l}\text { Force modulation etched silicon probe-type atomic force cantilever } \\
\text { (Nanosensors). Detected by the displacement of reflected beam }\end{array}$ \\
\hline Pavlov et al., 2009 & Glass microneedles inserted into myofibril & $\begin{array}{l}\text { Microneedle movement detected by linear photodiode array }(10,680 \\
\text { pixels) }\end{array}$ \\
\hline \multicolumn{3}{|l|}{ Single filaments } \\
\hline VanBuren et al., 1994a,b & Glass microneedle coated with HMM & Microneedle deflection detected by video microscopy \\
\hline $\begin{array}{l}\text { Holohan and Marston, } \\
2005\end{array}$ & Magnetic beads coated with gelsolin & Electromagnet and video microscopy \\
\hline Kaya et al., 2017 & $\begin{array}{l}\text { Myosin/rod copolymer on glass surface, actin-biotin phalloidin } \\
\text { TRITC attached to streptavidin coated bead }\end{array}$ & $\begin{array}{l}\text { Optical trap detected by feedback needed to keep bead steady under } \\
\text { load }\end{array}$ \\
\hline Pertici et al., 2018 & $\begin{array}{l}\text { HMM on glass microfiber coated with nitrocellulose, actin attached } \\
\text { to gelsolin coated bead }\end{array}$ & $\begin{array}{l}\text { Optical trap detected by feedback needed to keep bead steady under } \\
\text { load }\end{array}$ \\
\hline Bing et al., 2000 & a-actinin attached to cover glass & Indirect: quantity of a-actinin that stops actin filament movement \\
\hline Cheng et al., 2020 & $\begin{array}{l}\text { Cantilever coated with a-actinin to specifically attach actin filament. } \\
\text { Myosin adheres spontaneously }\end{array}$ & Microfabricated silicon nitride cantilever bending \\
\hline
\end{tabular}
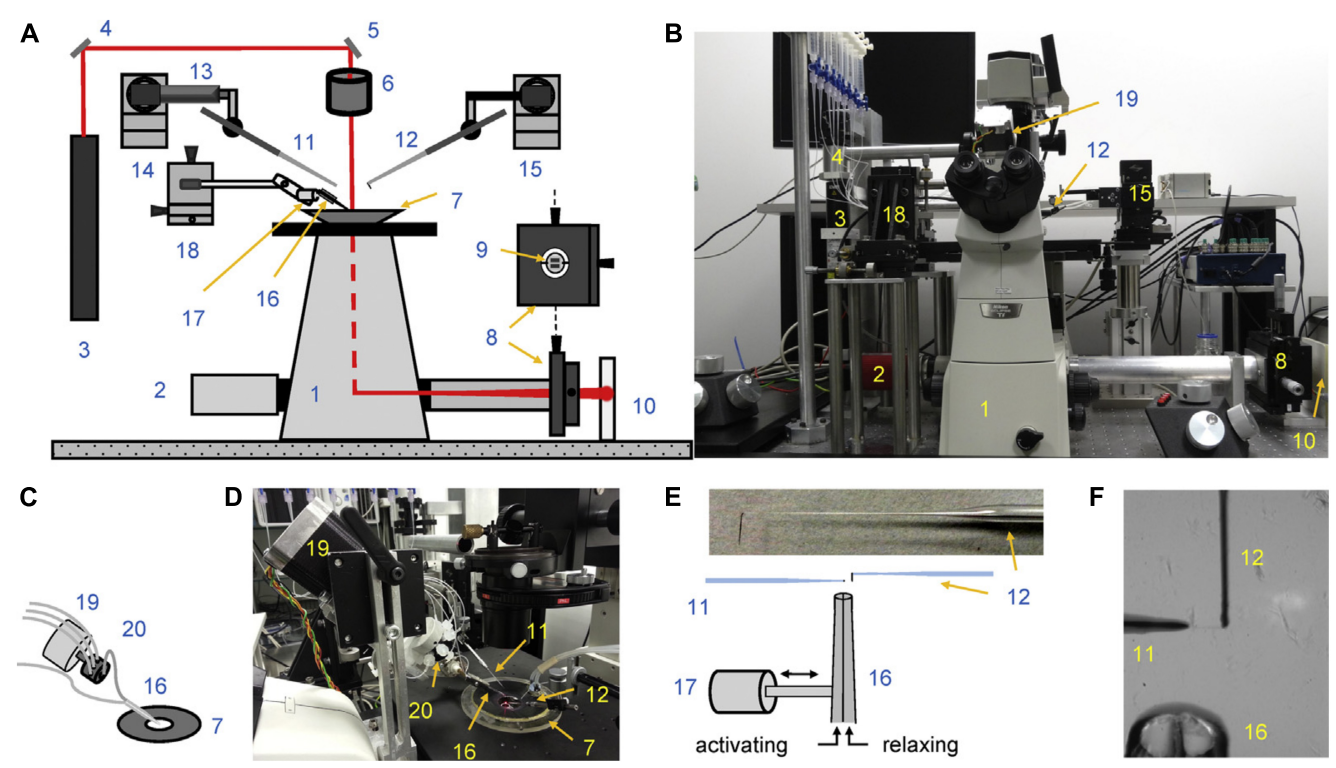

FIGURE 1 | Experimental setup. (A,B) Schematic overview (A) and photograph (B) show the key elements of the apparatus. (C,D) Schematics (C) and photograph (D) of multi-solution change system. (E) Schematics of cantilever and ultrafast solution change system. The left-sided microtool is relatively stiff and is mounted onto a piezo actuator for fast myofibril length change. The right-sided glass microtools is a high compliance L-shaped cantilever that acts as a force sensor. The inset shows a photograph showing the shape of the cantilever tip. (F) The photograph shows two microtools holding a small myofibril bundle. The relaxing and activating solutions are applied via the adjacent barrels of a double-barreled $(\mathrm{Q})$ micropipette. The numbers on the figure indicate: 1, inverted microscope; 2, camera; 3, laser; 4 and 5, mirrors; 6, microscope condenser; 7, bath mounted onto a microscope stage; 8, XY positioning stage; 9, segmented photodiode; 10, projection plane for visual control of cantilever positioning; 11, left microtool; 12, right microtool (cantilever force sensor); 13, piezo actuator; 14 and 15, left and right micromanipulators for the positioning of left and right microtool, respectively; 16, double-barreled Q pipette; 17, stepper motor controlling the position of the double-barreled Q pipette; 18, XYZ positioning stage for the ultrafast two solutions switching system; 19, stepper motor controlling the valve opening of the eight-channel valve; 20 , eight-channel valve. Reproduced from Vikhorev et al. (2015) with permission.

a close relationship of this process with the product release step of the crossbridge cycle (Tesi et al., 2002). The interpretation of the relaxation curve and its relationship to heterogeneous sarcomere lengthening was fully described by Stehle et al. (2009). Both $\mathrm{k}_{\mathrm{LIN}} / \mathrm{t}_{\mathrm{LIN}}$ and $\mathrm{k}_{\mathrm{REL}}$ are altered by physiological and pathological perturbations that affect thin filament relaxation rate such as cardiac TnI phosphorylation or HCM related mutations (Song et al., 2013; Vikhorev et al., 2014).

When fully implemented, the myofibril contractility method allows a wide range of measurement possibilities. Mechanical 

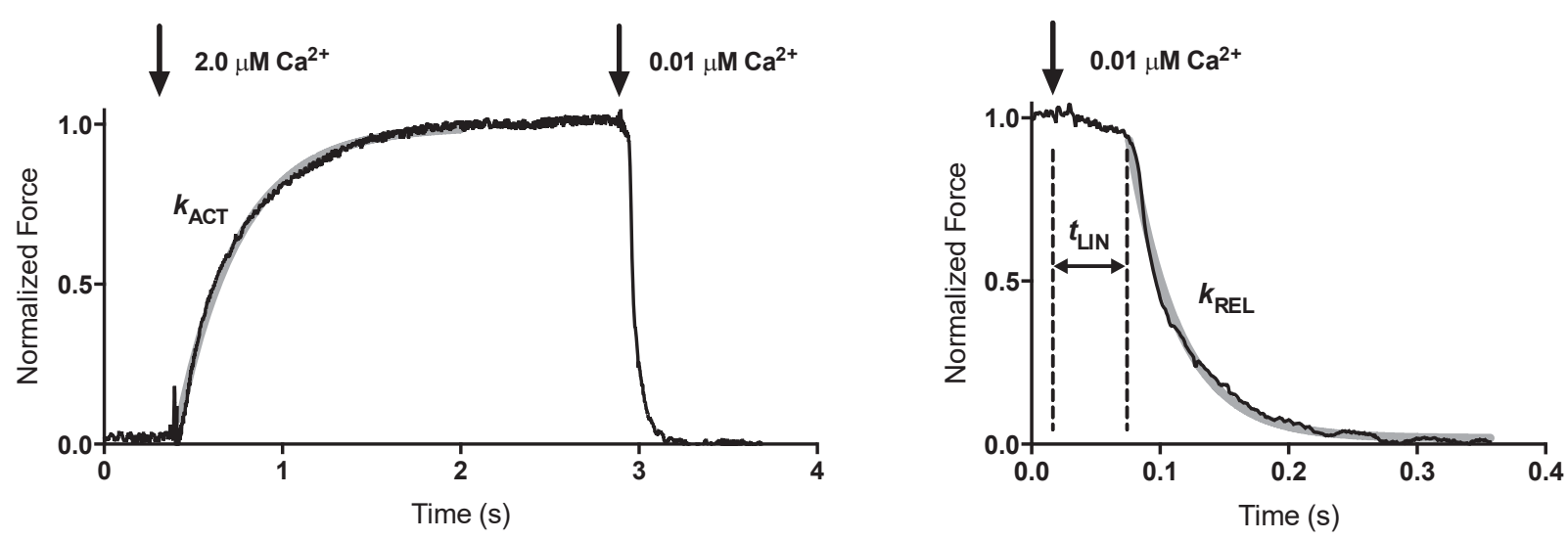

FIGURE 2 | Measurement of contractility in single mouse cardiac muscle myofibrils. Time course of contraction and relaxation. The arrows indicate the time when the solutions were switched. The kinetic parameters maximum force $\left(F_{\max }\right)$, rate of force development $\left(\mathrm{k}_{\mathrm{ACT}}\right)$, duration $\left(\mathrm{t}_{\mathrm{LIN}}\right)$ and slope $\left(\mathrm{k}_{\mathrm{LIN}}\right)$ of the slow relaxation phase, and rate of the fast relaxation phase $\left(k_{R E L}\right)$ were determined from the traces. The slow-relaxation phase was counted from the initiation of micropipette movement. The beginning of the exponential phase of relaxation was considered as the end of slow-relaxation phase. The relaxation phase is shown over an expanded timescale to show the initial linear, nearly isometric force decay period tLIN, followed by an exponential relaxation with a rate constant kREL. The gray solid lines represent the best exponential fits to the data. The temperature was $17^{\circ} \mathrm{C}$. Reproduced from Vikhorev et al. (2014) with permission.

kinetics similar to whole muscle fiber measurements are possible with myofibrils as demonstrated by the quick release/restretch protocol used to measure $\mathrm{k}_{\mathrm{tr}}$ (Figure $\mathbf{3 A}$ ). It is feasible to reproduce most of the mechanical perturbation protocols that have been used in chemically skinned fibers, but in practice these have not been extensively explored since they do not differ greatly whilst the opportunities for transient biochemical perturbation that is possible in myofibril studies yields exciting new insights. Passive stiffness can also be measured and provides results that are wholly due to sarcomeric elements, mainly titin (Linke et al., 1997; Makarenko et al., 2004; Opitz et al., 2004; Figure 3C). Solution changing can be adapted to measure response to a range of concentrations and generate dose-response curves (Figure 3B). A number of unique complex protocols are also accessible, for instance the simulation of the heart work cycle by combining carefully timed $\mathrm{Ca}^{2+}$ jumps up and down with length ramps simulating "systolic" shortening and "diastolic" lengthening between isometric phases (Figure 3D). The addition of video microscopy allows sarcomere length to be measured, enabling accurate sarcomere length adjustments, for instance when measuring length-dependent activation. During relaxation these observations have shown transient heterogeneous shortening of sarcomeres in a myofibril which has important implications for the structural changes behind the relaxation process (Stehle et al., 2002, 2009).

\section{APPLICATIONS}

Because the myofibril measurements can be made with any suitable source of muscle the methodology has been applied to many systems. The dynamics of $\mathrm{Ca}^{2+}$ activation of force, defined by $\mathrm{F}_{\max }, \mathrm{k}_{\mathrm{ACT}}$, $\mathrm{t}_{\mathrm{LIN}}, \mathrm{k}_{\mathrm{LIN}}$, and $\mathrm{k}_{\mathrm{REL}}$ and $\mathrm{Ca}^{2+}$ activation curves have been measured in myofibrils from both skeletal and cardiac muscles. Studies include:

(1) Comparing wild type with mutant muscles from transgenic mice such as the ACTC mutations E99K (HCM) and E361G (DCM) (Song et al., 2013; Vikhorev et al., 2014) or in mutant human heart myofibrils such as MYH7 R403Q, TNNT2 K280N, and titin truncating mutations (Ferrantini et al., 2009; Piroddi et al., 2019; Vikhorev et al., 2020).

(2) Based on techniques developed in skinned fibers (Brenner et al., 1999), troponin or tropomyosin can be exchanged with recombinant troponin components such as the HCM mutations TNNI3 R145G and TNNT2 K280N (Kruger et al., 2005; Piroddi et al., 2019) or tropomyosin isoforms or mutations (Siththanandan et al., 2009; Janco et al., 2012; Nixon et al., 2013; Scellini et al., 2014). Such studies have clearly demonstrated the effects of mutations on $\mathrm{Ca}^{2+}$ sensitivity and on the kinetic parameters.

(3) The same methodology can be applied to the effects of contractile protein phosphorylation, notably by PKA, on the $\mathrm{Ca}^{2+}$ sensitivity and relaxation kinetics (Vikhorev et al., 2014, 2020) and to determine the effects of myofilament targeting drugs such as Mavacamten and Omecamtiv Mecarbil (Scellini et al., 2020, 2021).

(4) The myofibril force assay is particularly useful when used with myofibrils from non-muscle sources. For instance in the study of embryonic heart, biopsies, cultured myocytes and particularly iPSC (Pioner et al., 2015, 2016; Racca et al., 2015; Iorga et al., 2018).

(5) A novel application is in measuring the effects of muscle stretch on passive elasticity and on active force generation. The former allows for assessment of titin mechanical properties (Linke et al., 1997; Makarenko et al., 2004; 


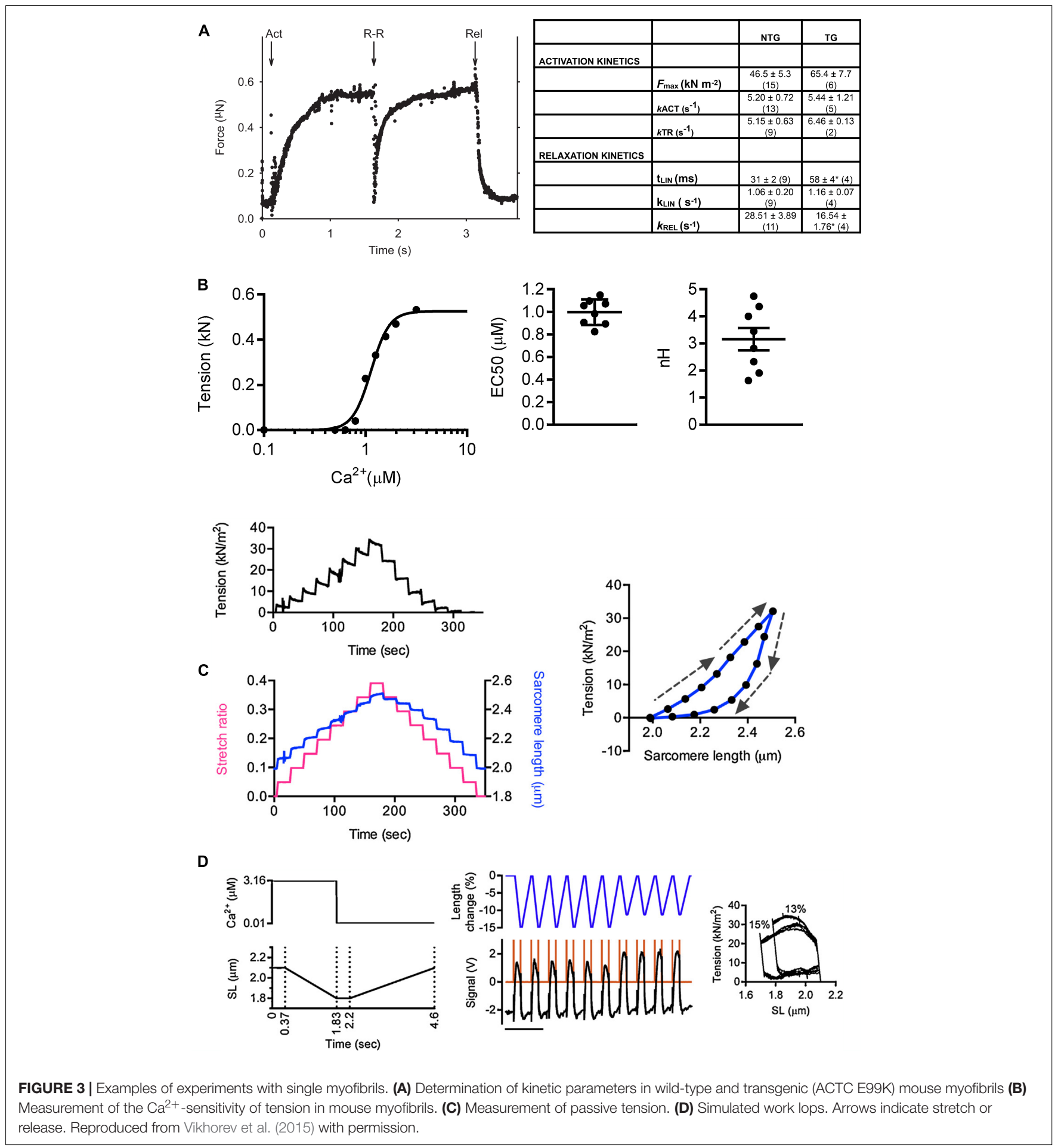

Opitz et al., 2004; Vikhorev et al., 2017) whilst the latter allows measurement of length dependent activation. Such studies have confirmed that both PKA-dependent phosphorylation and titin mutations can modulate length dependent activation whist having little or no effect on baseline contractility (Vikhorev et al., 2020).
(6) The single myofibril force assay has been adapted to measure force from a single sarcomere or even halfsarcomeres. Contraction-relaxation cycles, a force-velocity relationship and even Length dependent activation was successfully measured in single sarcomeres (Pavlov et al., 2009; Minozzo et al., 2013; Lowey et al., 2018). 
A

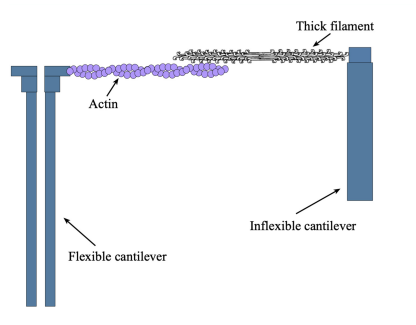

B

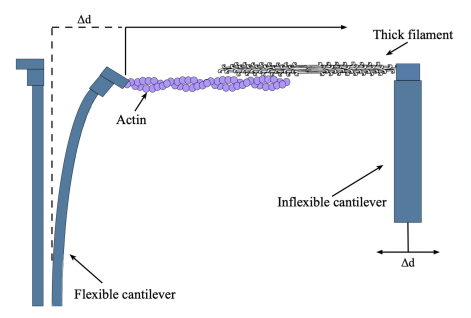

C

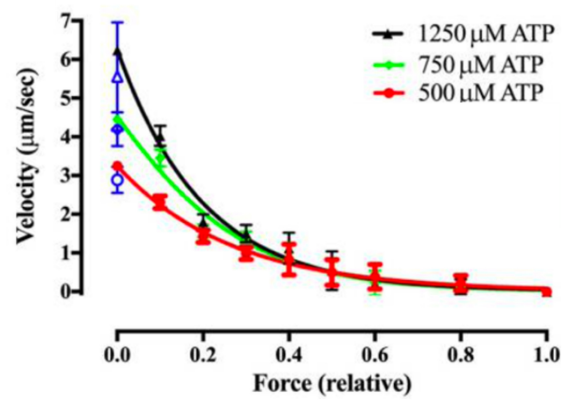

FIGURE 4 | The microfabricated cantilever single filament force assay. (A,B) Schematic view of the microfabricated cantilever experimental setup. Thick filament and actin filaments were attached to the cantilevers and brought into contact. The filaments y produce a force that causes the displacement of the flexible cantilever, which enabled force to be measured. (C) Example of a single filament force -velocity plot. Figures provided by Drs Y-S Sheng and DE Rassier.

A

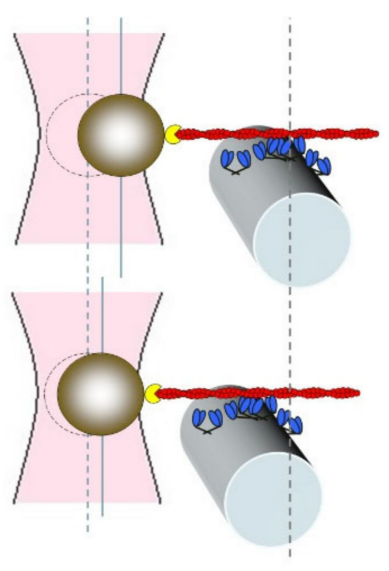

C

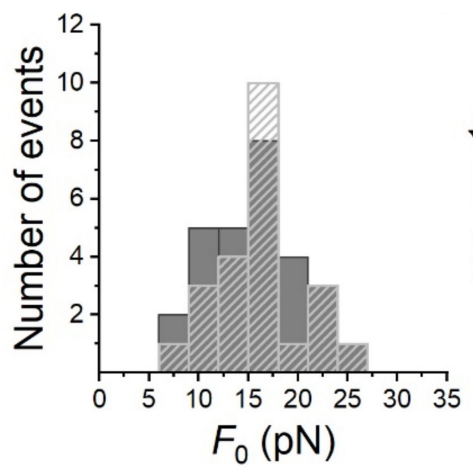

B

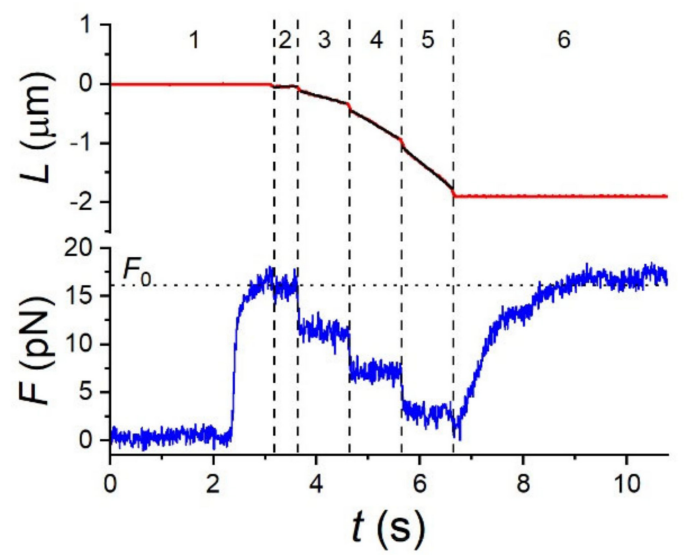

D

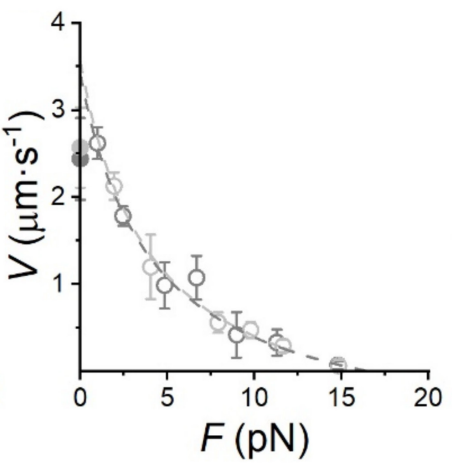

E

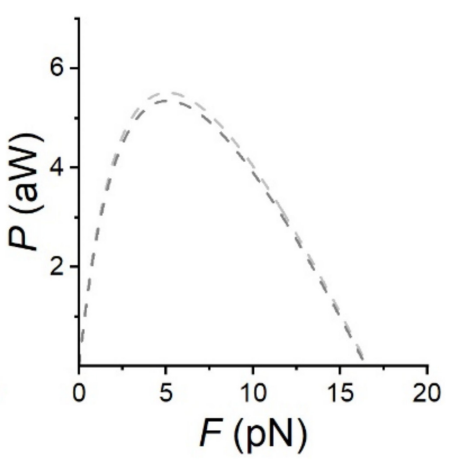

FIGURE $\mathbf{5}$ | Performance of the rabbit HMM-based nanomachine. (A) Schematic representation of two snapshots during the interaction between the actin filament and the motor ensemble. Upper panel: in position clamp at FO; lower panel: in force clamp at 0.4 FO. (B) Recording of the actin filament sliding (L, upper trace, red) and force ( $F$, lower trace, blue) during an interaction. Numbers bounded by dashed lines identify the different time intervals (it) as detailed in the text. (C) Frequency distribution of FO. Data are plotted in classes of $3 \mathrm{pN}$; dark gray bars, measurements in $0.1 \mathrm{mM} \mathrm{CaCl} 2$ ( $=77 \mathrm{M}$ free $\left[\mathrm{Ca}^{2+}\right]$ ); light gray dashed bars, in the absence of $\mathrm{Ca}^{2+}$. (D) F-V relation in $0.1 \mathrm{mM} \mathrm{CaCl}_{2}$ (dark gray open circles) and in $\mathrm{Ca}^{2+}$-free solution (light gray open circles). Points are mean $\pm \mathrm{SD}$ from individual experiments, grouped in classes of force 0.15 FO wide. Dark and light gray filled symbols on the ordinate are the Vf in the in vitro motility assay (IVMA) on rabbit HMM with and without $\mathrm{Ca}^{2+}$, respectively. The dashed lines are Hill's hyperbolic equation fits to the data with the same color code as symbols. Data in c and d are from 28 experiments in $0.1 \mathrm{mM} \mathrm{CaCl}_{2}$ and 23 experiments in $\mathrm{Ca}^{2+}$-free solution. (E) Power (P) versus F, calculated from the corresponding F-V fits in (D), with the same color code. Reproduced from Pertici et al. (2020) with permission. 


\section{SINGLE FILAMENT FORCE MEASUREMENTS}

Measurement of contractility at the single filament level enables us to determine the characteristics of the actomyosin contractile machine in the absence of the effects of structure of the sarcomere. To go to singe filaments requires different, more sensitive techniques, generally derived from in vitro motility assay or optical trap methodology (see Table 1).

The in vitro motility assay was devised about 30 years ago (Kron et al., 1991) and has been immensely successful for assaying the unloaded contractility of muscle filaments, characterizing myosins and thin filament regulatory proteins and their posttranslational modifications (Fraser and Marston, 1995; Gordon et al., 1997; Marston, 2003; Batters et al., 2014).

Adaptation of the IVMA to measure force requires a probe, usually attached to the thin filament, acting on immobilized myosin. In the first studies this was simply a bed of HMM molecules as used in normal IVMA but more sophisticated setups using whole thick filaments and introducing filament orientation have been devised.

The first studies used microneedles attached to thin filaments using NEM-HMM for attachment, developed by Kishino and Yanagida (1988) and Chaen et al. (1989). These were far more compliant than used for myofibrils since the forces obtained are in the region of $50 \mathrm{pN}$. Video microscopy is used to watch the needle bend until it stalls. The angle then represents the isometric force which is generally expressed as $\mathrm{pN} / \mu \mathrm{m}$ length of the actin filament overlap; values in the region of $10 \mathrm{pN} / \mu \mathrm{m}$ actin are obtained. VanBuren successfully used this technique to determine the effects of light chain removal and of myosin and tropomyosin isoform switches on force generation (VanBuren et al., 1994a,b, 1999). Homsher et al. (2000) used this technique to demonstrate $\mathrm{Ca}^{2+}$ control of force in single regulated thin filaments.

A more recent version of this methodology uses microfabricated AFM cantilevers as the tension probe (Figure 4). The stiffness of the cantilever is $0.18 \mathrm{pN} / \mathrm{nm}$. This study also used whole thick filaments in the correct orientation for optimum interaction with thin filaments. These measurements obtained values between 60 and $100 \mathrm{pN} / \mu \mathrm{m}$ which may more closely indicate the real maximum force generated by myosin motors. Since the inflexible cantilever can be moved to compensate for muscle shortening it is possible to make further measurements, such as a forcevelocity plot, which closely resembles that of intact muscle (Cheng et al., 2020).

Another method used for imposing load on a single actin filament is by magnetic force. The actin filaments are attached to magnetic beads via gelsolin in a normal IVMA cell and an electromagnet applies a magnetic force. Bead-tailed filaments moving over a bed of HMM slowed down under an increasing magnetic load, eventually stalled and then slid backward under increasing load before detaching from the surface. Singlefilament force-velocity curves were constructed and a stalling force of about $600 \mathrm{pN} / \mu \mathrm{m}$ of actin filament estimated. This method has not seen much use due to the very low yield of viable bead-tailed filaments (Bershitsky et al., 1996; Hamelink, 1999; Holohan and Marston, 2005).

A more widely used methodology for measuring force in single filaments is the indirect method, based on an internal load imposed on actin filaments by an anchored actin binding protein in a conventional in vitro motility assay. Warshaw et al. (1990) first demonstrated this principle using N-ethylmaleimide (NEM)-treated heavy meromyosin (HMM) and p-phenylenedimaleimide (pPDM)-treated HMM, while Janson et al. (1992) showed that a-actinin and filamin can also stop filament movement. The greater the myosin motor force on an actin filament, the higher the concentration of actin-binding protein needed to stop movement. Bing and Marston first demonstrated that the effect of a-actinin on the fraction of filaments moving is a consistent and sensitive method that can detect changes in isometric force of single actin filaments interacting with immobilized HMM (Bing et al., 2000). The initial studies investigated how tropomyosin and troponin may control force but only measured relative force, however, an analysis of how retardation works by Greenberg and Moore (2010) showed that it was possible to extract quantitative data from such measurements.

The optical trap has been used for many years to measure very small movements and has been mainly applied to single molecule studies of unloaded contractility. To measure force needs a much stiffer optical trap which can be limiting (Veigel et al., 1998, 2003). Recently the optical trap method has been used for force measurements of the interaction of actin with a small ensemble of myosin molecules. There is a trade-off here between the maximum amount of force the trap can handle and the minimum number of myosin-actin interactions needed for continuous movement. Skeletal muscle has a low "duty ratio" which means that if the steady-state number of interactions is too low the filaments can detach from the actin at physiological ATP concentrations. Kaya et al. (2017) used whole myosin filaments, but in order to reduce the interactions to not overload the optical trap the filaments were copolymers of myosin and myosin rod (1:200 ratio) and the estimated steady-state number of interactions was just 37 in a $600 \mathrm{~nm}$ myosin filament. Pertici et al. (2020) coated HMM onto a microfiber at an appropriate dilution that enabled a minimum of 32 steady state interaction (Figure 5). Pertici et al. suggest the geometry of their setup is superior to that of Kaya et al. (2017) since the actin and HMM can be adjusted to exert force in a linear rather than oblique direction and because the HMM, being concentrated on a small surface, has a constant area of actin-myosin overlap unlike the two-filament system where the length of overlap is a variable. On the other hand, the two-filament system using myosin filaments does have the correct orientation of myosin heads whereas in the "nanomachine" HMM is randomly orientated toward actin.

Both these methods found that myosin acts in a coordinated way when interacting with a single actin filament; maximum isometric force and a force-velocity curve were obtained (see Figure 5). 
In general, single filament systems offer a potential tool for investigating muscle contractile and regulatory protein mechanisms and the effects of mutations and post-translational modifications on force production that could match the equivalent unloaded studies using the in vitro motility assay. So far such studies have been limited to the thick filament (Rassier and Leite, 2020; Pertici et al., 2021) with only one study using single filaments to study thin filament regulation (Ishii et al., 2019).

\section{CONCLUSION}

Over the last 20 years, techniques for measuring force in single myofibrils or myofilaments have increasingly been used for fundamental studies of striated muscle contractile mechanisms and their regulation by $\mathrm{Ca}^{2+}$, post translational modifications and disease-related mutations. The techniques are exacting, and throughput is low but the results are accurate and provide detailed information of contractility at the subcellular level that cannot be obtained by any other method.

The key advantages of subcellular contractility measurement are twofold. Firstly, the contractile apparatus is directly studied

\section{REFERENCES}

Ayittey, P. N., Walker, J. S., Rice, J. J., and de Tombe, P. P. (2009). Glass microneedles for force measurements: a finite-element analysis model. Pflugers Arch. 457, 1415-1422. doi: 10.1007/s00424-008-0605-3

Bartoo, M. L., Popov, V. I., Fearn, L. A., and Pollack, G. H. (1993). Active tension generation in isolated skeletal myofibrils. J. Musc. Res. Cell Motily 14, 498-510. doi: 10.1007/bf00297212

Batters, C., Veigel, C., Homsher, E., and Sellers, J. R. (2014). To understand muscle you must take it apart. Front. Physiol. 5:90. doi: 10.3389/fphys.2014.00090

Bershitsky, S., Tsaturyan, A. K., Bershitskaya, O. N., Blange, T., and Van Kaam, F. A. M. (1996). ${ }^{\star}$ Electromagnetic device for loading molecular motors. J. Muscle Res. Cell Motil. 17, 156.

Bing, W., Knott, A., and Marston, S. (2000). A simple method for measuring the relative force exerted by myosin on actin filaments in the in vitro motility assay: evidence that tropomyosin and troponin increase force in single filaments. Biochem. J. 350, 693-699. doi: 10.1042/0264-6021:3500693

Brenner, B., Kraft, T., Yu, L. C., and Chalovich, J. M. (1999). Thin filament activation probed by fluorescence of $\mathrm{N}$-((2-(Iodoacetoxy)ethyl)-Nmethyl)amino-7-nitrobenz-2-oxa-1, 3-diazole-labeled troponin I incorporated into skinned fibers of rabbit psoas muscle. Biophys. J. 77, 2677-2691. doi: 10.1016/s0006-3495(99)77102-x

Chaen, S., Oiwa, K., Shimmen, T., Iwarmoto, I., and Sugi, H. (1989). Simultaneous recordings of force and sliding movements between myosin coated glass microneedle and actin cables in vitro. Proc. Natl. Acad. Sci. U.S.A. 86, 15101514. doi: 10.1073/pnas.86.5.1510

Cheng, Y.-S., Leite, F. D. S., and Rassier, D. E. (2020). The load dependence and the force-velocity relation in intact myosin filaments from skeletal and smooth muscles. Am. J. Physiol. 318, C103-C110. doi: 10.1152/ajpcell.00339. 2019

Colomo, F., Nencini, S., Piroddi, N., Poggesi, C., and Tesi, C. (1998). Mechanisms of work production and work absorption in muscle. Adv. Exp. Med. Biol. 453, 373-382. doi: 10.1007/978-1-4684-6039-1 42

Colomo, F., Piroddi, N., Poggesi, C., Kronnie, G. T., and Tesi, C. (1997). Active and passive forces of isolated myofibrils from cardiac and fast skeletal muscle of the frog. J. Physiol. 500, 535-548. doi: 10.1113/jphysiol.1997.sp022039

Ferrantini, C., Belus, A., Piroddi, N., Scellini, B., Tesi, C., and Poggesi, C. (2009). Mechanical and energetic consequences of HCM-causing and secondly, rapid solution changing permits the measurement of the kinetics of the force changes induced by $\mathrm{Ca}^{2+}$ concentration changes for the first time.

These methods have potential for investigating regulation, post translational modifications and mutations in a huge variety of systems. The versatility of these systems plus the ability to combine force measurements with optical measurements allows the possibility of new types of experiments not yet envisaged. For instance, single myofibril and myofilament data can be valuable to form the basis of multiscale modeling of muscle (Månsson, 2019, 2020).

\section{AUTHOR CONTRIBUTIONS}

The author confirms being the sole contributor of this work and has approved it for publication.

\section{FUNDING}

This work was supported by the BHF project Grant PG/17/5/32705 to Petr Vikhorev and SM.

mutations. J. Cardiovasc. Trans. Res. 2, 441-451. doi: 10.1007/s12265-0099131-8

Fraser, I. D., and Marston, S. B. (1995). In vitro motility analysis of actintropomyosin regulation by troponin and calcium. The thin filament is switched as a single cooperative unit. J. Biol. Chem. 270, 7836-7841. doi: 10.1074/jbc.270. 14.7836

Friedman, A. L., and Goldman, Y. E. (1996). Mechanical characterization of skeletal muscle myofibrils. Biophys. J. 71, 2774-2785. doi: 10.1016/s0006-3495(96) 79470-5

Gordon, A. M., LaMadrid, M. A., Chen, Y., Luo, Z., and Chase, P. B. (1997). Calcium regulation of skeletal muscle thin filament motility in vitro. Biophys. J. 72, 1295-1307. doi: 10.1016/S0006-3495(97)78776-9

Greenberg, M. J., and Moore, J. R. (2010). The molecular basis of frictional loads in the in vitro motility assay with applications to the study of the loaded mechanochemistry of molecular motors. Cytoskeleton (Hoboken, NJ) 67, 273-285. doi: 10.1002/cm.20441

Hamelink, W. (1999). Muscle Contraction At the Molecular Level: Actin Myosin Interaction Studied in An in Vitro Motility Assay. Amsterdam: University of Amsterdam. .

Holohan, S.-J. P., and Marston, S. B. (2005). Force-velocity relationship of single actin filament interacting with immobilised myosin measured by electromagnetic technique. IEE Proc. Nanobiotechnol. 152, 113-120. doi: 10. 1049/ip-nbt:20045003

Homsher, E., Lee, D. M., Morris, C., Pavlov, D., and Tobacman, L. S. (2000). Regulation of force and unloaded sliding speed in single thin filaments: effects of regulatory proteins and calcium. J. Physiol. (Lond) 524(Pt 1), 233-243. doi: 10.1111/j.1469-7793.2000.00233.x

Iorga, B., Schwanke, K., Weber, N., Wendland, M., Greten, S., Piep, B., et al. (2018). Differences in contractile function of myofibrils within human embryonic stem cell-derived cardiomyocytes vs. adult ventricular myofibrils are related to distinct sarcomeric protein isoforms. Front. Physiol. 8:1111. doi: 10.3389/fphys. 2017.01111

Ishii, S., Suzuki, M., Ishiwata, S. I, and Kawai, M. (2019). Functional significance of HCM mutants of tropomyosin, V95A and D175N, studied with in vitro motility assays. Biophys. Physicobiol. 16, 28-40. doi: 10.2142/biophysico.16.0_28

Iwazumi, T. (1987). High-speed ultrasensitive instrumentation for myofibril mechanics measurements. Am. J. Physiol. 252(Pt 1), C253-C262. doi: 10.1152/ ajpcell.1987.252.2.C253 
Janco, M., Kalyva, A., Scellini, B., Piroddi, N., Tesi, C., Poggesi, C., et al. (2012). $\alpha$-Tropomyosin with a D175N or E180G mutation in only one chain differs from tropomyosin with mutations in both chains. Biochemistry 51, 9880-9890. doi: 10.1021/bi301323n

Janson, L. W., Sellers, J. R., and Taylor, D. L. (1992). Actin-binding proteins regulate the work performed by myosin II motors on single actin filaments. Cell Motil. Cytoskeleton 22, 274-280. doi: 10.1002/cm.970220407

Johns, E. C., Ryder, K. O., Hodson, E. A., Hart, G., Mulligan, I. P., Lipscomb, S., et al. (1999). Investigating the relaxation rate, following diazo-2 photolysis, of a skinned trabecular preparation from guinea-pig hypertrophied left ventricle. Pflugers Arch. 438, 771-777. doi: 10.1007/s004249900131

Kaya, M., Tani, Y., Washio, T., Hisada, T., and Higuchi, H. (2017). Coordinated force generation of skeletal myosins in myofilaments through motor coupling. Nat. Comm. 8:16036. doi: 10.1038/ncomms16036

Kishino, A., and Yanagida, T. (1988). Force measurements by micromanipulation of a single actin filament by glass needles. Nature 334, 74-76. doi: 10.1038/ $334074 \mathrm{a} 0$

Kron, S. J., Toyoshima, Y. Y., Uyeda, T. Q., and Spudich, J. A. (1991). Assays for actin sliding movement over myosin-coated surfaces. Methods Enzymol. 196, 399-416. doi: 10.1016/0076-6879(91)96035-p

Kruger, M., Zittrich, S., Redwood, C., Blaudeck, N., James, J., Robbins, J., et al. (2005). Effects of the mutation R $145 \mathrm{G}$ in human cardiac troponin I on the kinetics of the contraction-relaxation cycle in isolated cardiac myofibrils. J. Physiol. 564(Pt 2), 347-357. doi: 10.1113/jphysiol.2004.079095

Linke, W. A., Ivemeyer, M., Labeit, S., Hinssen, H., Rüegg, J. C., and Gautel, M. (1997). Actin-titin interaction in cardiac myofibrils: probing a physiological role. Biophys. J. 73, 905-919. doi: 10.1016/S0006-3495(97)78123-2

Lowey, S., Bretton, V., Joel, P. B., Trybus, K. M., Gulick, J., Robbins, J., et al. (2018). Hypertrophic cardiomyopathy $\mathrm{R} 403 \mathrm{Q}$ mutation in rabbit $\beta$-myosin reduces contractile function at the molecular and myofibrillar levels. Proc. Natl. Acad. Sci. U.S.A. 115, 11238-11243. doi: 10.1073/pnas.1802967115

Makarenko, I., Opitz, C. A., Leake, M. C., Neagoe, C., Kulke, M., Gwathmey, J. K., et al. (2004). Passive stiffness changes caused by upregulation of compliant titin isoforms in human dilated cardiomyopathy hearts. Circ. Res. 95, 708-716. doi: 10.1161/01.RES.0000143901.37063.2f

Månsson, A. (2019). Comparing models with one versus multiple myosin-binding sites per actin target zone: the power of simplicity. J. Gen. Physiol. 151, 578-592. doi: 10.1085/jgp.201812301

Månsson, A. (2020). Hypothesis: single actomyosin properties account for ensemble behavior in active muscle shortening and isometric contraction. Int J. Mol. Sci. 21:8399. doi: 10.3390/ijms21218399

Marston, S. (2003). Random walks with thin filaments: application of in vitro motility assay to the study of actomyosin regulation. J. Musc. Res. Cell Motil. 24, 149-156. doi: 10.1023/a:1026097313020

Minozzo, F. C., Baroni, B. M., Correa, J. A., Vaz, M. A., and Rassier, D. E. (2013). Force produced after stretch in sarcomeres and half-sarcomeres isolated from skeletal muscles. Nat. Sci. Rep. 3:2320. doi: 10.1038/srep02320

Nixon, B. R., Liu, B., Scellini, B., Tesi, C., Piroddi, N., Ogut, O., et al. (2013). Tropomyosin Ser-283 pseudo-phosphorylation slows myofibril relaxation. Arch. Biochem. Biophys. 535, 30-38. doi: 10.1016/j.abb.2012.11.010

Opitz, C. A., Leake, M. C., Makarenko, I., Benes, V., and Linke, W. A. (2004). Developmentally regulated switching of titin size alters myofibrillar stiffness in the perinatal heart. Circ. Res. 94, 967-975. doi: 10.1161/01.RES.0000124301. 48193.E1

Pavlov, I., Novinger, R., and Rassier, D. E. (2009). The mechanical behavior of individual sarcomeres of myofibrils isolated from rabbit psoas muscle. Am. J. Physiol. 297, C1211-C1219. doi: 10.1152/ajpcell.00233.2009

Pertici, I., Bianchi, G., Bongini, L., Cojoc, D., Taft, M. H., Manstein, D. J., et al. (2021). Muscle myosin performance measured with a synthetic nanomachine reveals a class-specific Ca2+-sensitivity of the frog myosin II isoform. J. Physiol. 599, 1815-1831. doi: 10.1113/jp280976

Pertici, I., Bianchi, G., Bongini, L., Lombardi, V., and Bianco, P. (2020). A Myosin II-based nanomachine devised for the study of Ca2+-Dependent mechanisms of muscle regulation. Int. J. Mol. Sci. 21:7372. doi: 10.3390/ijms2119 7372

Pertici, I., Bongini, L., Melli, L., Bianchi, G., Salvi, L., Falorsi, G., et al. (2018). A myosin II nanomachine mimicking the striated muscle. Nat. Comm. 9:3532. doi: 10.1038/s41467-018-06073-9
Pioner, J. M., Pioner, J. M., Yang, K.-C., Pabon, L., Racca, A. W., Jeong, M. Y., et al. (2015). Cell and myofibril contractile properties of hiPSC-Derived cardiomyocytes from a patient with a MYH7 mutation associated with familial cardiomyopathy. Biophys. J. 108(Suppl. 1):201a. doi: 10.1016/j.bpj.2014.11.1110

Pioner, J. M., Racca, A. W., Klaiman, J. M., Yang, K.-C., Guan, X., Pabon, L., et al. (2016). Isolation and mechanical measurements of myofibrils from human induced pluripotent stem cell-derived cardiomyocytes. Stem Cell Rep. 6, 885-896. doi: 10.1016/j.stemcr.2016.04.006

Piroddi, N., Witjas-Paalberends, E. R., Ferrara, C., Ferrantini, C., Vitale, G., Scellini, B., et al. (2019). The homozygous $\mathrm{K} 280 \mathrm{~N}$ troponin T mutation alters crossbridge kinetics and energetics in human HCM. J. Gen. Physiol. 151, 18-29. doi: 10.1085/jgp.201812160

Racca, A. W., Klaiman, J. M., Pioner, J. M., Cheng, Y., Beck, A. E., MoussaviHarami, F., et al. (2015). Contractile properties of developing human fetal cardiac muscle. J. Physiol. 594, 437-452. doi: 10.1113/jp271290

Rassier, D. E., and Leite, F. D. S. (2020). Sarcomere length non-uniformity and force regulation in myofibrils and sarcomeres. Biophys. J. 119, 2372-2377. doi: 10.1016/j.bpj.2020.11.005

Scellini, B., Piroddi, N., Dente, M., Ferrantini, C., Coppini, R., Poggesi, C., et al. (2020). Impact of mavacamten on force generation in single myofibrils from rabbit psoas and human cardiac muscle. Biophys. J. 118:7a. doi: 10.1016/j.bpj. 2019.11.231

Scellini, B., Piroddi, N., Dente, M., Vitale, G., Coppini, R., Ferrantini, C., et al. (2021). Omecamtiv mecarbil modulation of force generation in single myofibrils from human cardiac muscle. Biophys. J. 120:249a. doi: 10.1016/j.bpj. 2020.11.1623

Scellini, B., Piroddi, N., Flint, G. V., Regnier, M., Poggesi, C., and Tesi, C. (2014). Impact of tropomyosin isoform composition on fast skeletal muscle thin filament regulation and force development. J. Musc. Res. Cell Motil. 36, 1-13. doi: 10.1007/s10974-014-9394-9

Siththanandan, V. B., Tobacman, L. S., Gorder, N. V., and Homsher, E. (2009). Mechanical and kinetic effects of shortened tropomyosin reconstituted into myofibrils. Pflugers Arch. : Eur. J. physiol. 458, 761-776. doi: 10.1007/s00424009-0653-3

Song, W., Vikhorev, P. G., Kashyap, M. N., Rowlands, C., Ferenczi, M. A., Woledge, R. C., et al. (2013). Mechanical and energetic properties of papillary muscle from ACTC E99K transgenic mouse models of hypertrophic cardiomyopathy. Am. J. Physiol. Heart Circ. Physiol. 304, H1513-H1524. doi: 10.1152/ajpheart.00951. 2012

Stehle, R., Kruger, M., and Pfitzer, G. (2002). Force kinetics and individual sarcomere dynamics in cardiac myofibrils after rapid $\mathrm{ca}(2+)$ changes. Biophys. J. 83, 2152-2161. doi: 10.1016/S0006-3495(02)73975-1

Stehle, R., Solzin, J., Iorga, B., and Poggesi, C. (2009). Insights into the kinetics of Ca2+-regulated contraction and relaxation from myofibril studies. Pflugers Arch. 458, 337-357. doi: 10.1007/s00424-008-0630-2

Tesi, C., Piroddi, N., Colomo, F., and Poggesi, C. (2002). Relaxation kinetics following sudden $\mathrm{Ca}(2+)$ reduction in single myofibrils from skeletal muscle. Biophys. J. 83, 2142-2151. doi: 10.1016/S0006-3495(02)73974-X

VanBuren, P., Palmiter, K. A., and Warshaw, D. M. (1999). Tropomyosin directly modulates actomyosin mechanical performance at the level of a single actin filament. Proc. Natl. Acad. Sci. U.S.A. 96, 12488-12493. doi: 10.1073/pnas.96. 22.12488

VanBuren, P., Waller, G. S., Harris, D. E., Trybus, K. M., Warshaw, D. M., and Lowey, S. (1994a). The essential light chain is required for full force production by skeletal muscle myosin. Proc. Natl. Acad. Sci. U.S.A. 91, 12403-12407. doi: 10.1073/pnas.91.26.12403

VanBuren, P., Work, S. S., and Warshaw, D. M. (1994b). Enhanced force generation by smooth muscle myosin in vitro. Proc. Natl. Acad. Sci. U.S.A. 91, 202-205.

Veigel, C., Bartoo, M. L., White, D. C. S., Sparrow, J. C., and Molloy, J. E. (1998). The stiffness of rabbit skeletal actomyosin cross-bridges determined with an optical tweezers transducer. Biophys. J. 75, 1424-1438. doi: 10.1016/s00063495(98)74061-5

Veigel, C., Molloy, J. E., Schmitz, S., and Kendrick-Jones, J. (2003). Load-dependent kinetics of force production by smooth muscle myosin measured with optical tweezers. Nat. Cell Biol. 5, 980-986. doi: 10.1038/ncb1060

Vikhorev, P., Ferenczi, M. A., and Marston, S. (2015). Instrumentation to study myofibril mechanics from static to artificial simulations of cardiac cycle. Methods X 3, 156-170. doi: 10.1016/j.mex.2016.02.006 
Vikhorev, P. G., Smoktunowicz, N., Munster, A. B., Copeland, O. X. N., Kostin, S., Montgiraud, C., et al. (2017). Abnormal contractility in human heart myofibrils from patients with dilated cardiomyopathy due to mutations in TTN and contractile protein genes. Sci. Rep. 7:14829. doi: 10.1038/s41598-017-13675-8

Vikhorev, P. G., Song, W., Wilkinson, R., Copeland, O., Messer, A. E., Ferenczi, M. A., et al. (2014). The dilated cardiomyopathy-causing mutation ACTC E361G in cardiac muscle myofibrils specifically abolishes modulation of $\mathrm{Ca}(2+)$ regulation by phosphorylation of troponin I. Biophys. J. 107, 2369-2380. doi: 10.1016/j.bpj.2014.10.024

Vikhorev, P. G., Vikhoreva, N. N., Yeung, W., Li, A., Lal, S., Remedios, C. G. D., et al. (2020). Titin-Truncating mutations associated with dilated cardiomyopathy alter length-dependent activation and its modulation via phosphorylation. Cardiovasc. Res. [Online ahead of print] cvaa316. doi: 10.1093/ $\mathrm{cvr} / \mathrm{cvaa} 316$

Warshaw, D. M., Desrosiers, J. M., Work, S. S., and Trybus, K. M. (1990). Smooth muscle myosin cross-bridge interactions modulate actin filament sliding velocity in vitro. J. Cell Biol. 111, 453-463. doi: 10.1083/jcb.111.2.453
Conflict of Interest: The author declares that the research was conducted in the absence of any commercial or financial relationships that could be construed as a potential conflict of interest.

Publisher's Note: All claims expressed in this article are solely those of the authors and do not necessarily represent those of their affiliated organizations, or those of the publisher, the editors and the reviewers. Any product that may be evaluated in this article, or claim that may be made by its manufacturer, is not guaranteed or endorsed by the publisher.

Copyright (c) 2022 Marston. This is an open-access article distributed under the terms of the Creative Commons Attribution License (CC BY). The use, distribution or reproduction in other forums is permitted, provided the original author(s) and the copyright owner(s) are credited and that the original publication in this journal is cited, in accordance with accepted academic practice. No use, distribution or reproduction is permitted which does not comply with these terms. 